\title{
Retrograde tracing with recombinant rabies virus reveals correlations between projection targets and dendritic architecture in layer 5 of mouse barrel cortex
}

\author{
DeLaine D. Larsen; lan R. Wickersham and Edward M. Callaway* \\ Systems Neurobiology Laboratories, The Salk Institute for Biological Studies, Neurosciences Graduate Program, University of California, \\ San Diego, La Jolla, CA, USA
}

Edited by: Rafael Yuste, Columbia University, USA

Reviewed by: Michael Brecht, Humboldt University of Berlin, Germany

Dmitri 'Mitya' Chklovskii, Cold Spring Harbor Laboratory, USA

\begin{abstract}
A recombinant rabies virus was used as a retrograde tracer to allow complete filling of the axonal and dendritic arbors of identified projection neurons in layer 5 of mouse primary somatosensory cortex (S1) in vivo. Previous studies have distinguished three types of layer 5 pyramids in S1: tall-tufted, tall-simple, and short. Layer 5 pyramidal neurons were retrogradely labeled from several known targets: contralateral S1, superior colliculus, and thalamus. The complete dendritic arbors of labeled cells were reconstructed to allow for unambiguous classification of cell type. We confirmed that the tall-tufted pyramids project to the superior colliculus and thalamus and that short layer 5 pyramidal neurons project to contralateral cortex, as previously described. We found that tall-simple pyramidal neurons contribute to corticocortical connections. Axonal reconstructions show that corticocortical projection neurons have a large superficial axonal arborization locally, while the subcortically projecting neurons limit axonal arbors to the deep layers. Furthermore, reconstructions of local axons suggest that tall-simple cell axons have extensive lateral spread while those of the short pyramids are more columnar. These differences were revealed by the ability to completely label dendritic and axonal arbors in vivo and have not been apparent in previous studies using labeling in brain slices.
\end{abstract}

Keywords: local circuits, pyramidal neuron, axonal branching, barrel cortex, rabies virus, thalamus, superior colliculus

\section{INTRODUCTION}

Information processing in the neocortex requires the precise connectivity of the underlying cortical circuitry. Individual cortical neurons make precise connections at many levels, including the extrinsic targets to which they project, the laminar specificity of their local axonal arbors, and the specific cell types to which they connect. For any given cortical neuron type, the choices that are made at each of these levels of specificity are correlated, presumably to match the local computations within a given cortical area to the computational needs of the distant structures that receive the cortical output. Here we focus on layer 5 pyramidal neurons, which provide a major cortical output and can be grouped into distinct cell types.

Previous work in our laboratory has distinguished three types of pyramidal neurons in layer 5 of mouse primary somatosensory cortex (S1) based on axonal and dendritic morphology: tall-tufted,

*Correspondence: Edward M. Callaway, Systems Neurobiology Laboratories, The Salk Institute for Biological Studies, La Jolla, CA 92037, USA; Neurosciences Graduate Program, University of California, San Diego, La Jolla, CA 92039, USA. e-mail: callaway@ salk.edu

TPresent address: DeLaine D. Larsen, Center for Neuroscience, University of California, Davis, Davis, CA 95616, USA.

:Present address: Ian R. Wickersham, Department of Brain and Cognitive Sciences, Massachusetts Institute of Technology, Cambridge, MA 02139, USA.

Received: 24 0ctober 2007; paper pending published: 26 November 2007; accepted: 11 February 2008; published online: 28 March 2008.

Citation: Front. Neural Circuits (2008) 1: 5. doi: 10.3389/neuro.04.005.2007

Copyright (c) 2008 Larsen, Wickersham and Callaway. This is an open-access article subject to an exclusive license agreement between the authors and the Frontiers Research Foundation, which permits unrestricted use, distribution, and reproduction in any medium, provided the original authors and source are credited. tall-simple, and short (Larsen and Callaway, 2006). In vitro labeling studies (Larsen and Callaway, 2006) have shown that two of the cell types, the tall-simple and short layer 5 pyramidal neurons, have similar laminar specificity of their local axonal arborizations, with arbors in both the deep and superficial layers. In contrast, the tall-tufted layer 5 pyramidal neurons have axonal arborizations mainly in the deep cortical layers. Other studies have shown that the tall-tufted layer 5 pyramidal neurons project subcortically to targets such as the superior colliculus and thalamus (Bourassa et al., 1995; Deschenes et al., 1994; Games and Winer, 1988; Hallman et al., 1988; Hubener and Bolz, 1988; Hubener et al., 1990; Killackey et al., 1989; Larkman and Mason, 1990; Tsiola et al., 2003; Veinante et al., 2000; Wise and Jones, 1977). The short layer 5 pyramidal neurons have been shown to be corticocortical projection neurons (Games and Winer, 1988; Hallman et al., 1988; Hubener and Bolz, 1988; Hubener et al., 1990; Larkman and Mason, 1990). The tallsimple layer 5 pyramidal neurons have an unknown projection target, but have been described before (Akemann et al., 2004; Chagnac-Amitai et al., 1990; Kim and Connors, 1993; Tsiola et al., 2003).

We were interested in better understanding the relationships between local axonal projections, extrinsic projections and anatomical cell types. For example, is the presence of an apical dendritic tuft (tall simple and tall-tufted cells) predictive of connections with subcortical structures? Or is there instead a correlation between the pattern of local axonal arbors (e.g., projections to superficial layers) and extrinsic connections? Furthermore, are there more subtle differences between the axonal arbors of different cell types that are not apparent from the limited axonal reconstructions achieved with in vitro labeling? Understanding these correlations is crucial to understanding the unique role of each layer 5 pyramidal cell type in cortical and subcortical information processing. 
We have recently described a recombinant rabies virus, $\mathrm{SAD} \Delta \mathrm{G}$-EGFP, that functions as an almost ideal retrograde tracer (Wickersham et al., 2007). Like naturally occurring rabies virus, it infects via axon terminals; however, because its envelope glycoprotein gene has been deleted, it is unable to spread transsynaptically, instead acting as a first-order retrograde tracer that labels only cells projecting to an injection site. Because the deleted gene has been replaced with the coding sequence for EGFP, these infected cells express vast amounts of EGFP, permitting the easy resolution of fine anatomical details (Wickersham et al., 2007).

Here we have used this recombinant rabies virus to retrogradely label layer 5 pyramidal neurons from three known projection targets: contralateral $\mathrm{S} 1$, superior colliculus, and thalamus. We find that the local axonal arborization pattern correlates with extrinsic projection target: both the tall-simple and the short pyramidal neurons have local projections to layer $2 / 3$ and extrinsic projections to other cortical areas, while the tall-tufted pyramidal neurons have local projections in deep layers and extrinsic projections to subcortical targets. Furthermore, the ability to label more extensive local axons in vivo than in brain slices revealed a difference between tall-simple and short layer 5 pyramidal neurons in their local axonal projections to superficial layers. The tall-simple neurons appear to have a patchy projection with greater lateral spread in superficial layers compared to a more columnar projection of short pyramidal neurons.

\section{MATERIALS AND METHODS}

\section{Animals}

C57BL6 mice were obtained from Harlan and kept on a 12-hour light/ dark cycle. All animals were treated in accordance with institutional and $\mathrm{NIH}$ guidelines for the Care and Use of Laboratory Animals.

\section{Virus injections}

C57BL6 mice were anesthetized with ketamine $(100 \mathrm{mg} / \mathrm{kg} \mathrm{IM})$ and xylazine $(10 \mathrm{mg} / \mathrm{kg} \mathrm{IM})$. Virus was loaded into pulled glass pipettes (tip inner diameter of 30-50 $\mu \mathrm{m}$ ) and injected using a Picospritzer III (Parker Hannifin/General Valve Corporation, Fairfield, NJ) at approximately $20 \mathrm{nl} /$ minute. $180-600 \mathrm{nl}$ of virus (7.5E7-2.5E8 infectious units) was injected at the following locations, with stereotaxic coordinates in millimeters relative to bregma: Barrel cortex: $-1.7 \mathrm{AP},+3 \mathrm{LM},-1.5 \mathrm{DV}$. Superior colliculus: $-3.64 \mathrm{AP},+1.0 \mathrm{LM},-1.75 \mathrm{DV}$. Thalamus: $-2.06 \mathrm{AP}$, $+1.125 \mathrm{LM},-3 \mathrm{DV}$. Virus production and titering was as previously described (Wickersham et al., 2007).

Mice were deeply anesthetized 7 days postinjection with $4 \%$ isoflurane and perfused transcardially with $4 \%$ paraformaldehyde in PBS. Brains were postfixed and cryopreserved overnight in $4 \%$ paraformaldehyde $/ 30 \%$ sucrose in PBS, then kept in $30 \%$ sucrose in PBS until sectioning. Brains were sectioned in the coronal plane at 100 um on a freezing microtome.

\section{Immunohistochemistry}

Immunohistochemistry for GFP was used to visualize the neuronal morphology and results in brighter, more stable labeling than the native GFP to allow for reconstructions. The sections were incubated in blocking solution (10\% NGS, 2\% BSA, 0.25\% Triton-X 100, $0.1 \mathrm{M} \mathrm{PB}$ ) for 2 hours. Sections were then incubated in a polyclonal anti-GFP (1:1000, Molecular Probes, Eugene, OR) overnight at $4^{\circ} \mathrm{C}$. The primary antibody was visualized with a Cy3-conjugated secondary antibody (1:100; Chemicon, Temecula, CA). After staining, the sections were counterstained with DAPI (10 uM, Sigma) to allow for the determination of laminar and areal borders. Sections were then mounted onto subbed slides, dehydrated, and coverslipped with Krystalon mounting medium (Fisher).

\section{Data analysis}

Neurons were reconstructed using an Olympus fluorescence microscope in concert with a Neurolucida computerized reconstruction system (MicroBrightfield, Williston, VT). First, low power (10× objective, $0.5 \mathrm{NA}$ ) maps were drawn of each selected section. Maps included locations of the cell bodies of all labeled cells, laminar borders, and borders of barrel cortex. The dendritic arbors of selected cells were then reconstructed at higher power (60× oil immersion objective, $1.40 \mathrm{NA}$ ) without the knowledge of cortical layers. After completion, the neuronal reconstructions were overlaid onto the low power maps, containing the cortical layers, for analysis. Each neuron was analyzed for the number of dendritic branch points per layer using a custom designed MatLab-based program. Total apical dendritic length was obtained in the analysis portion of the NeuroExplorer software package (MicroBrightfield, Williston, VT).

Only animals with injection sites, identified by the presence of labeled glia, that were clearly within the correct structure, were included in the analysis. Examples of injection sites for each of the targets are found in Figure 1. Reconstructed neurons came from at least 2 separate animals for each injected structure and all neurons were located in the barrel field of primary somatosensory cortex, identified by cell dense barrel septa. The neurons were classified as either tall-tufted, tall-simple, or short pyramidal neurons based on the criteria identified in our previous quantitative
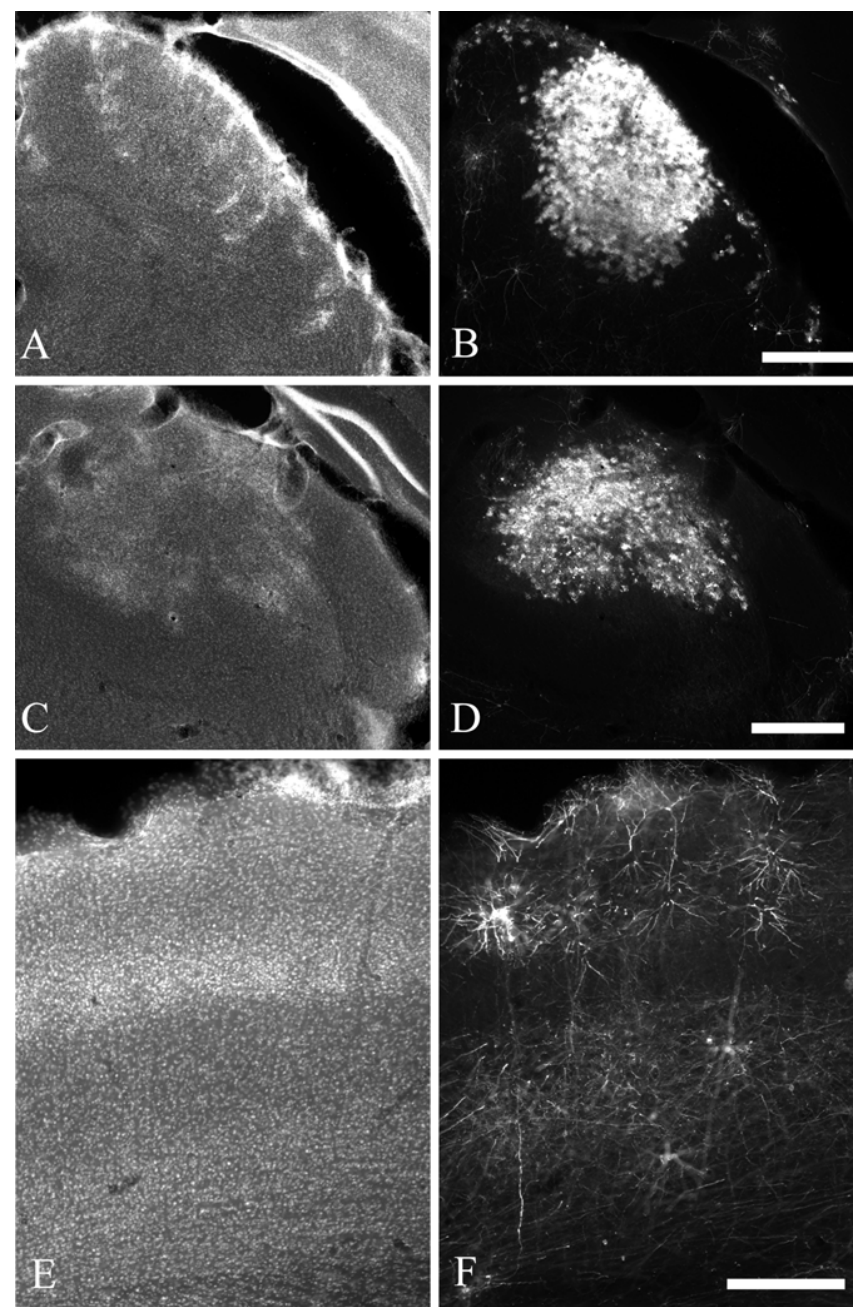

Figure 1. Photomicrographs illustrating examples of virus injection sites, visualized by immunohistochemistry for EGFP (B, D, F). The injections are identified by the large number of labeled glia, which can also be seen in the DAPI counterstain $(\boldsymbol{A}, \boldsymbol{C}, \mathbf{E})$ as patches of brightly labeled cells. The injection sites illustrated are only from one section; most sites extended across 5-10 sections in the anterior-posterior axis. For the images of the superior colliculus $(\boldsymbol{A}, \boldsymbol{B})$ and thalamus $(\boldsymbol{C}, \boldsymbol{D})$ the midline is to the right and dorsal is to the top. For the images of cortex $(\boldsymbol{E}, \boldsymbol{F})$ the midline is to the right and the pial surface is to the top. The scale bar in panel $\boldsymbol{D}$ is 500 um and applies to panels $\boldsymbol{A}, \boldsymbol{B}, \boldsymbol{C}$, and $\boldsymbol{D}$. The scale bar in panel $\boldsymbol{F}$ is 250 um and applies to panels $\boldsymbol{E}$ and $\boldsymbol{F}$. 

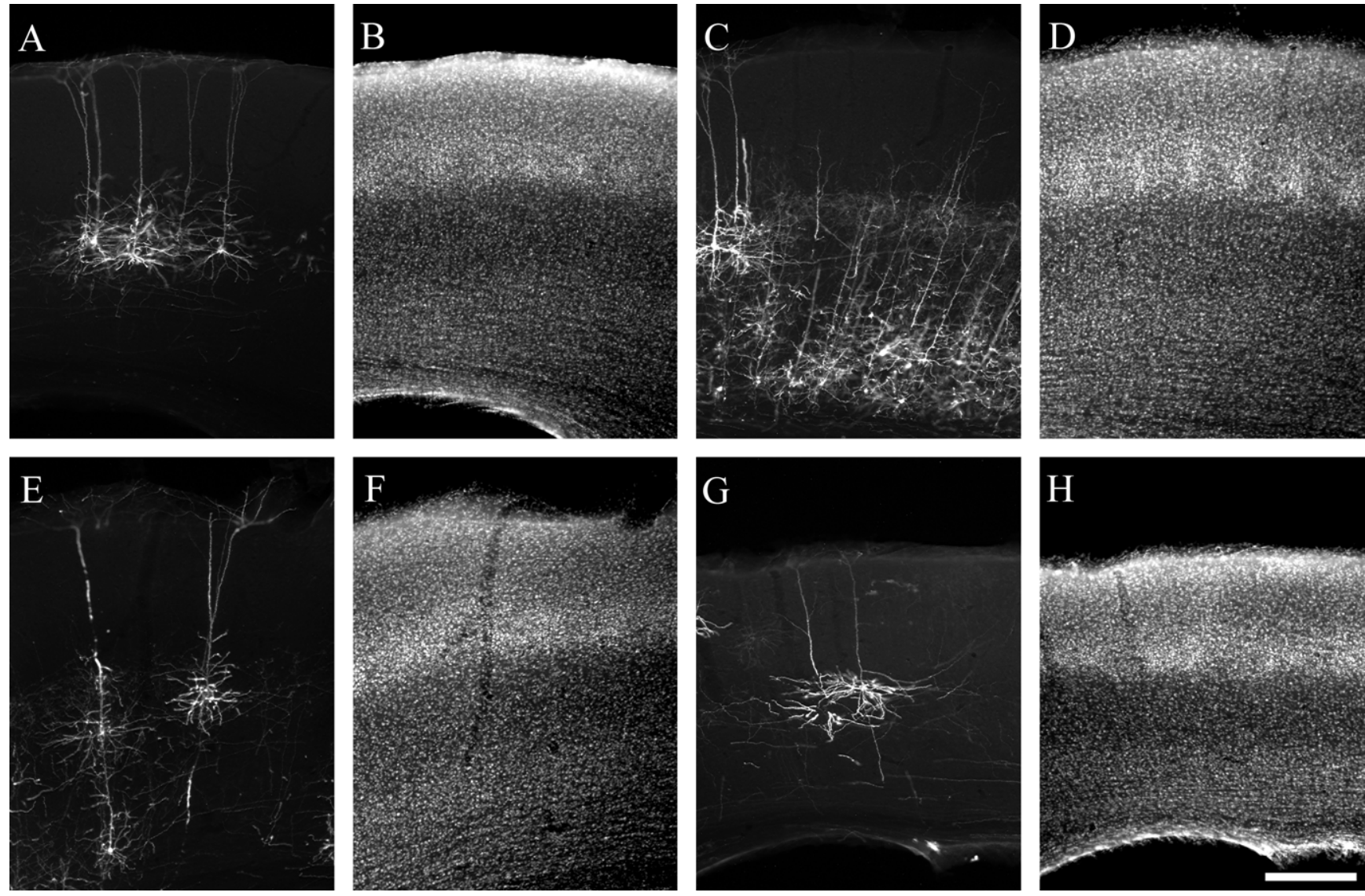

Figure 2. Photomicrographs illustrating typical labeling quality of pyramidal neurons using a glycoprotein-deleted rabies virus expressing EGFP. Pyramidal neurons are visualized by immunohistochemistry for EGFP and a Cy3-conjugated secondary antibody $(\boldsymbol{A}, \mathbf{C}, \boldsymbol{E}, \mathbf{G})$ and laminar borders are determined by a DAPI counterstain $(\mathbf{B}, \mathbf{D}, \mathbf{F}, \boldsymbol{H})$. For all images the pial surface is towards the top. Neurons are retrogradely labeled from injections in the superior colliculus $(\boldsymbol{A}, \mathbf{B})$, thalamus $(\mathbf{C}-\mathbf{F})$, and contralateral $S 1(\mathbf{G}, \mathbf{H})$. The neurons labeled from the superior colliculus are from the same animal as the example injection site in Figure $1 \mathbf{B}$. The thalamic injection for panel $C$ was too large to allow for reconstruction due to the density of label, but illustrates the difference in axonal arborizations with a large amount of axons present in the deep layers and almost no label in the superficial layers. The scale bar in $\mathrm{H}$ is 250 um and applies to all images.

study of these neurons labeled in brain slices (Larsen and Callaway, 2006). Dendritic arbors of a total of 30 layer 5 pyramidal neurons were reconstructed to allow for unambiguous classification: 13 corticocortical, 9 corticotectal, and 8 corticothalamic. We also reconstructed the axons for 6 of the neurons: 2 corticocortical tall-simple, 2 corticocortical short, 1 corticotectal tall-tufted and 1 corticothalamic tall-tufted.

Figures 1 and 2 were prepared by importing images from software provided with Optronics MicroFire camera (Optronics, Goleta, CA), into Adobe Photoshop and subsequently into Adobe Illustrator. Minimal alterations were made in the brightness and contrast of these images.

\section{RESULTS}

\section{Quality of neuronal label}

We utilized a recombinant rabies virus, $\mathrm{SAD} \Delta \mathrm{G}$-EGFP, as a retrograde tracer to label pyramidal neurons in layer 5 of the primary barrel field of S1. Many traditional retrograde tracing techniques are limited in their ability to completely label the neuronal morphology. Some groups have combined retrograde labeling with fluorescent dyes and subsequent intracellular fills to reveal the morphology of labeled neurons (e.g., Katz, 1987). The use of the SAD $\Delta$ G-EGFP rabies virus as a retrograde tracer has several advantages, including only being taken up by synaptic terminals at the injection site (not axons of passage), and having the ability to replicate intracellularly and express extremely high levels of EGFP (Wickersham et al., 2007). Furthermore, since labeling occurs in vivo, there is no loss of distant axonal processes as occurs during the preparation of brain slices for in vitro labeling. The SAD $\triangle$ G-EGFP rabies virus is unable to infect additional neurons transsynaptically and the EGFP expressed by the virus completely fills the neurons, allowing the visualization of both dendrites and axons (Figure 2).

\section{Quantitative comparisons of cell types}

In a previous study of the development of layer 5 pyramidal neurons (Larsen and Callaway, 2006) we identified objective criteria to distinguish between 3 distinct types of layer 5 pyramidal neurons. In that study, neurons were intracellularly labeled with biocytin in cortical slices and classified as tall-tufted, tall-simple, or short. Two main features could be used to reliably distinguish between cell types: the presence of an apical tuft within layer 1 and total apical dendritic length. The identification of short pyramids was most straightforward; the apical dendrite fails to reach layer 1 . Tall-simple and tall-tufted pyramids both have apical tufts in layer 1, but differ quantitatively in total apical dendritic length. Most notably, there is no overlap in the distributions of total apical dendritic length (Larsen and Callaway, 2006). The validity of this measure is reinforced by the observation that tall-simple layer 5 pyramidal neurons have axons projecting to superficial layers, while tall-tufted layer 5 pyramidal neurons do not.

In the present study we used these same criteria to unambiguously classify layer 5 pyramidal neurons. The quantitative comparisons of total 


\section{Corticotectal Neurons}
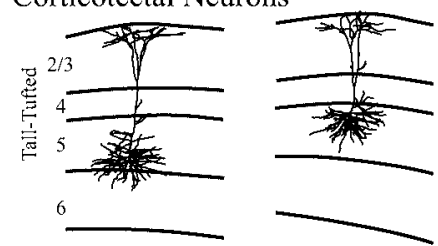

Corticothalamic Neurons
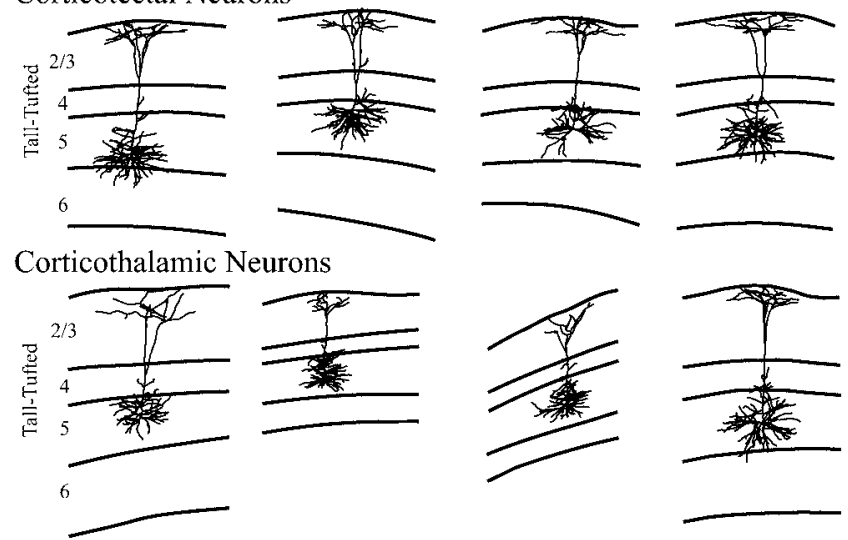

Corticocortical Neurons
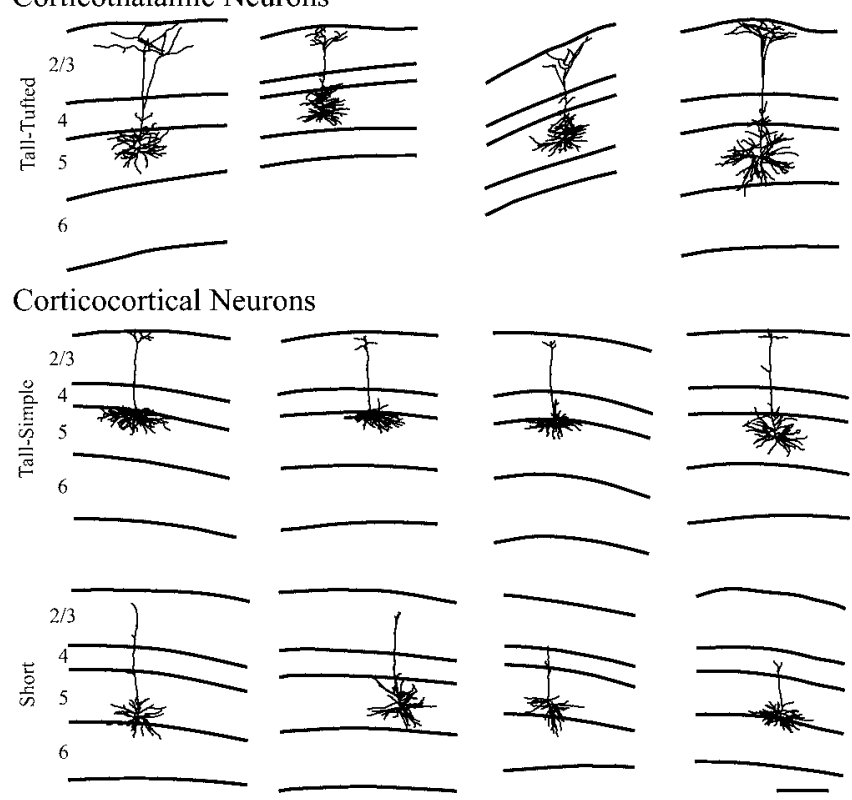

Figure 3. Representative examples of reconstructions of neurons from each injection site. Lines define the laminar boundaries for each cortical layer. The upper line indicates the top of the section, with layer 1 included with layer 2/3. Neurons are grouped according to injection site and cell type. The scale bar is 250 microns and applies to all drawings.

apical dendritic length for neurons with different extrinsic projections (see below) further reinforce the validity of these criteria. We also measured numbers of apical dendritic branches and report here on the utility of this measure for cell classification.

\section{Pyramidal neurons labeled from the superior colliculus}

We reconstructed dendritic arbors of 9 neurons from 3 mice with injections in the superior colliculus, spanning all the layers. All 9 neurons had the appearance of tall-tufted layer 5 pyramidal neurons (Figure 3 ). The talltufted morphology is characterized by a large cell body with an apical dendrite that extends to layer 1 and ends with a large apical dendritic tuft ending in layer 1 (Figure 3). These results correspond with previous studies using more traditional tracers (Games and Winer, 1988; Hallman et al., 1988; Hubener and Bolz, 1988; Hubener et al., 1990; Koester and O'Leary, 1992; Schofield et al., 1987; Tsiola et al., 2003; Wang and McCormick, 1993). These 9 neurons had apical dendritic lengths that ranged from 3.23 to $6.04 \mathrm{~mm}$ with an average of $4.79 \pm 0.33 \mathrm{~mm}$ (Figure $4 \mathrm{~A}$; mean $\pm \mathrm{SEM}$ ). The total number of apical dendritic branches ranged from 32 to 66 with an average of $49.22 \pm 3.89$ (mean \pm SEM), with the majority of branches occurring in layer $2 / 3(21.89 \pm 1.78$; mean \pm SEM; Figure $4 B)$ and layer 5 (24.78 \pm 3.20 ; mean \pm SEM; Figure $4 B)$.

\section{Pyramidal neurons labeled from the thalamus}

We reconstructed dendrites of 8 neurons from 2 mice with injections in the thalamus. All 8 neurons had the appearance of tall-tufted layer 5 pyramidal neurons with the same characteristics as the corticotectal neurons described above (Figure 3). Our thalamic injections were large,
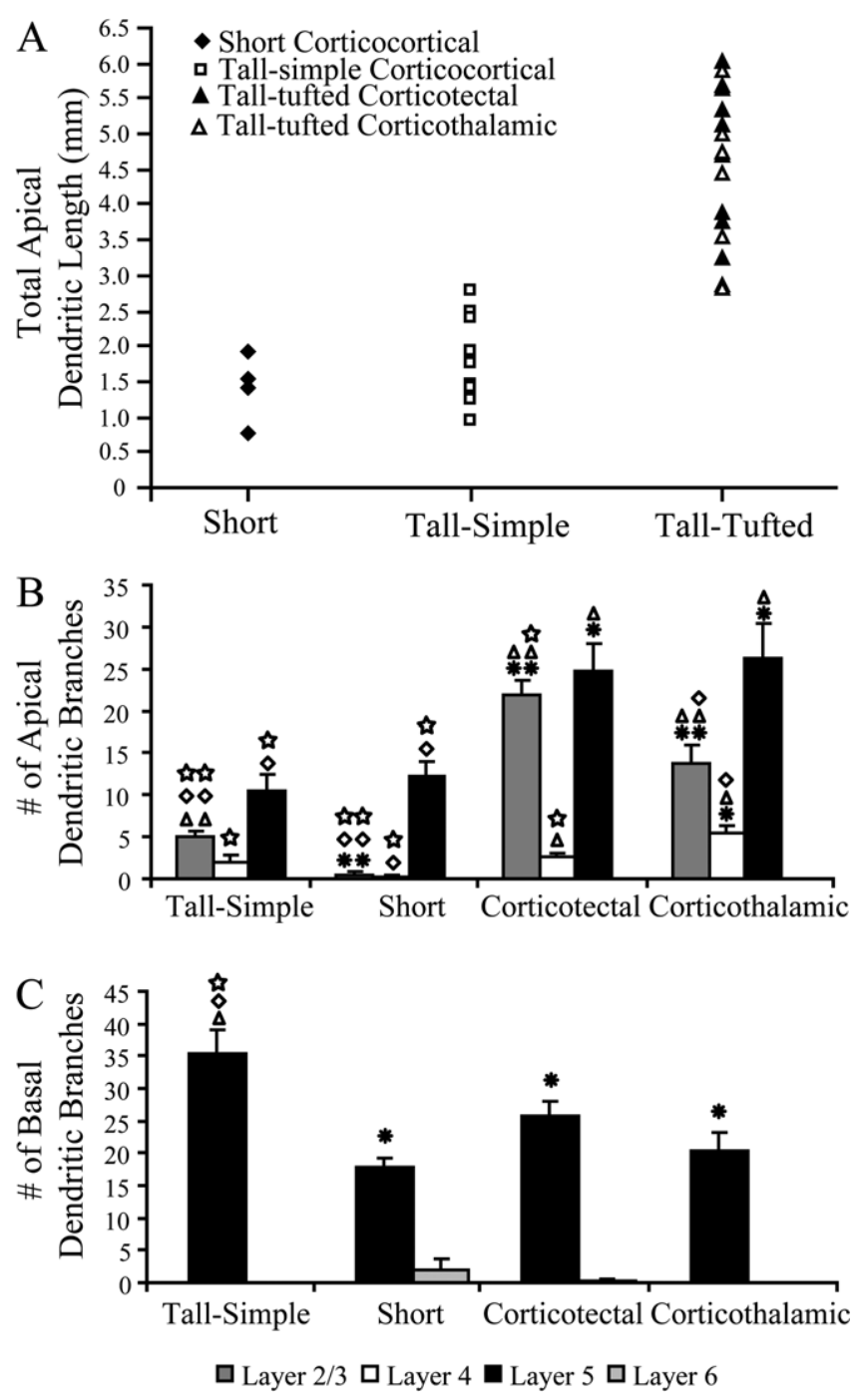

Figure 4. Distributions of total apical dendritic length for all layer 5 pyramidal neurons reconstructed, plotted by cell type. (A) The talltufted layer 5 pyramidal neurons have significantly greater total apical dendritic length than either the tall-simple or short layer 5 pyramidal neurons $(p<0.001 ; t$-test). Bar graphs indicate the number of apical dendritic branch points (B) and basal dendritic branch points (C) per cell in each cortical layer (mean \pm SEM). Asterisks denote significant difference from tall-simple neurons. Triangles denote significant difference from short neurons. Diamonds denote significant difference from tall-tufted corticotectal neurons. Squares denote significant difference from tall-tufted corticothalamic neurons. One symbol $=p<0.05, t$-test. Two symbols $=p<0.001, t$-test.

encompassing multiple nuclei, but both injections included the posterior group $(\mathrm{Po})$, the probable target of the neurons labeled in layer 5 (Bourassa et al., 1995).

These 8 neurons had apical dendritic lengths that ranged from 2.81 to $5.88 \mathrm{~mm}$ with an average of $4.25 \mathrm{~mm} \pm 0.38$ (Figure $4 \mathrm{~A}$; mean \pm SEM). The total number of apical dendritic branches ranged from 32 to 66 with an average of $45.50 \pm 3.94$ (mean \pm SEM), with the majority of branches occurring in layer $2 / 3(13.88 \pm 2.15$; mean $\pm S E M$; Figure $4 B)$ and layer $5(26.25 \pm 4.20$; mean $\pm \mathrm{SEM}$; Figure $4 \mathrm{~B})$. These values did not differ significantly from the SC-projecting neurons for either total apical dendritic length ( $p$-value $=0.3, t$-test) or number of apical dendritic branches ( $p$-value $=0.5, t$-test). Altogether the 17 tall-tufted neurons (SC- and thalamus-projecting) had apical dendritic length that ranged from 2.81 to 6.04 with an average of $4.54 \mathrm{~mm} \pm 0.25$ (mean \pm SEM), 
which is significantly greater $(p<0.001, t$-test) than either tall-simple or short layer 5 pyramidal neurons (see below). The total number of apical dendritic branches ranged from 32 to 66 with an average of $47.47 \pm 2.72$ (mean $\pm \mathrm{SEM}$ ), which is significantly greater $(p<0.001, t$-test) than either tall-simple or short layer 5 pyramidal neurons (see below).

\section{Pyramidal neurons labeled from contralateral S1}

We reconstructed dendrites of 13 neurons from 3 mice with injections in contralateral S1. We identified 2 types of pyramidal neurons from these cortical injections. Four of the 13 neurons were short layer 5 pyramidal neurons (Figure 3). The short layer 5 pyramidal neurons are characterized by smaller cell bodies and an apical dendrite that only extends into layer $2 / 3$ and has few if any branches in superficial layers. The short layer 5 pyramidal neurons have been identified in previous studies as corticocortical projection neurons (Games and Winer, 1988; Hallman et al., 1988; Hubener and Bolz, 1988; Hubener et al., 1990; Koester and 0'Leary, 1992). These 4 neurons had apical dendritic lengths that ranged from 0.76 to $1.91 \mathrm{~mm}$ with an average of $1.41 \mathrm{~mm} \pm 0.24$ (mean \pm SEM). The total number of apical dendritic branches ranged from 8 to 17 with an average of $13.00 \pm 1.96$ (mean $\pm S E M$ ), with the majority of branches occurring in layer 5 (12.25 \pm 1.75 ; mean \pm SEM; Figure 4B).

The remaining 9 of 13 neurons were identified as tall-simple layer 5 pyramidal neurons (Figure 3). The tall-simple layer 5 pyramidal neurons are characterized by having a smaller, more elongated cell body than
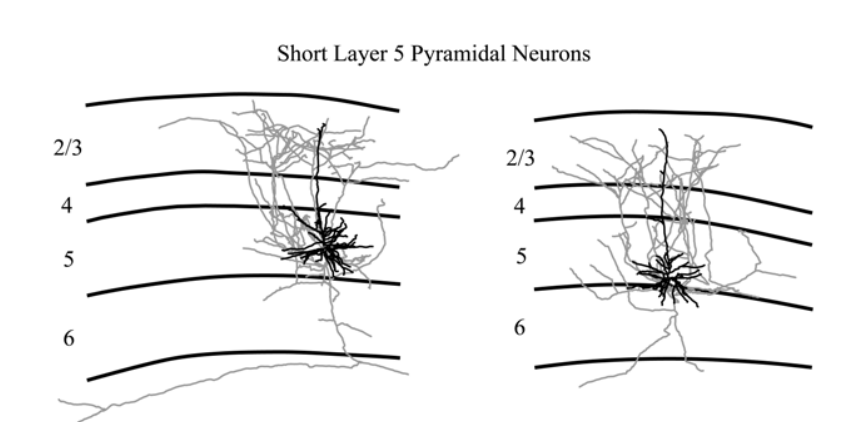

Tall-Simple Layer 5 Pyramidal Neurons
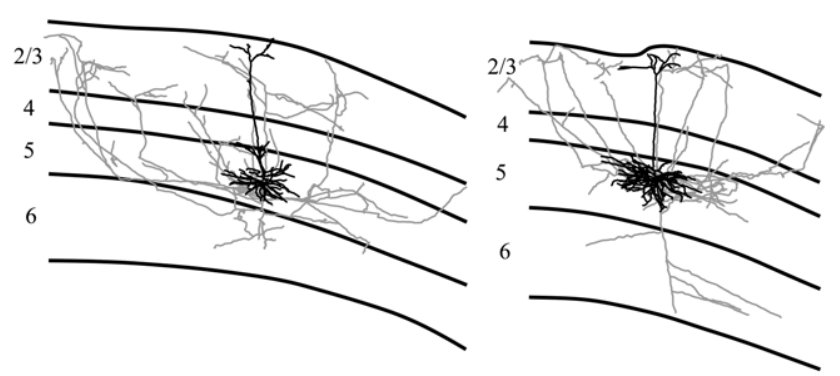

Tall-Tufted Layer 5 Pyramidal Neurons
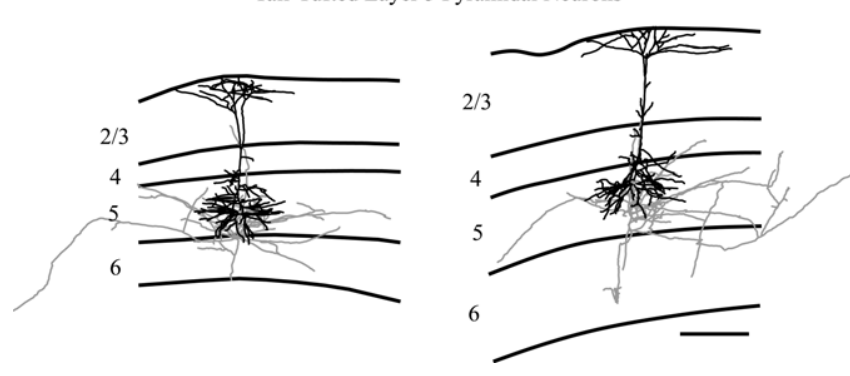

Figure 5. Representative examples of reconstructions of neurons from each injection site. Neurons are illustrated with axons depicted in gray and dendrites in black. Lines define the laminar boundaries for each cortical layer. The upper line indicates the top of the section, with layer 1 included with layer 2/3. Neurons are grouped according to cell type. The two short layer 5 pyramidal neurons illustrated are also included in Figure 3, without the axons included. The scale bar is 250 microns and applies to all drawings. the tall-tufted neurons and an apical dendrite that extends to layer 1, ending with a simple tuft. Previous studies that identified the short layer 5 pyramidal neurons as the source of the corticocortical projection neurons did not observe the tall-simple cell type. These 9 neurons had apical dendritic lengths that ranged from 0.95 to $2.77 \mathrm{~mm}$ with an average of $1.83 \mathrm{~mm} \pm 0.21$ (mean $\pm \mathrm{SEM}$ ). The total number of apical dendritic branches ranged from 10 to 26 with an average of $17.67 \pm 1.77$ (mean \pm SEM), with the majority of branches occurring in layer $5(10.56 \pm 2.01$; mean \pm SEM; Figure $4 \mathrm{~B})$ and a modest amount of branches in layer 2/3 (13.88 \pm 2.15 ; mean \pm SEM; Figure $4 B)$.

\section{Local axonal arbors}

To investigate the axonal arbors of each layer 5 pyramidal neuron type, axonal arbors were reconstructed for a subset of the neurons, including 2 neurons of each cell type. While this low $n$ precludes us from a meaningful quantitative analysis of the axonal morphologies, we would like to note that visual inspections of these axons (see Figure 5) are in excellent agreement with our previous report that tall-tufted layer 5 pyramidal neurons project local axons within deep cortical layers, while tall-simple and short layer 5 pyramidal neurons have extensive projections to superficial layers. However, the more complete reconstructions that were made possible by in vivo labeling revealed an unexpected difference in the axons of the short layer 5 pyramidal neurons in comparison with the tall-simple layer 5 pyramidal neurons. The axonal projections to the superficial layers differed: those of the two short layer 5 pyramidal neurons were relatively columnar, while the axons of the two tall-simple layer 5 pyramidal neurons had extensive laterally spreading and patchy axons within layer 2/3. This qualitative difference was not readily apparent in our sample of neurons labeled in vitro.

\section{DISCUSSION}

In a previous study examining the development of layer-specific axonal arborizations of pyramidal neurons in S1 of mice, we were able to distinguish 3 different pyramidal cell types in layer 5: tall-tufted, tall-simple, and short (Larsen and Callaway, 2006). The tall-simple layer 5 pyramidal neurons have not been well described in the literature and the projection target(s) of this cell type is unknown. We were interested in whether the tall-simple layer 5 pyramidal neurons have the same extrinsic connections as the tall-tufted or the short layer 5 pyramidal neurons. If the tall-simple layer 5 pyramidal neurons share projections with the tall-tufted layer 5 pyramidal neurons, then the dendritic morphology, with the presence of an apical dendritic tuft in layer 1 for both cell types, correlates with the projection target. If the tall-simple layer 5 pyramidal neurons share projections with the short layer 5 pyramidal neurons, then the axonal morphology, with the presence of axonal arbors in the superficial layers for both cell types, correlates with the projection target. Both possible results have implications for the roles different layer 5 pyramidal cell types play in cortical circuitry.

We found that the axonal morphology of the cell types correlates with the projection targets more than the dendritic morphology (Figure 6). For the thirty layer 5 pyramidal neurons reconstructed in the present study, the tall-simple and short layer 5 pyramidal neurons account for all of the corticocortical projection neurons while the tall-tufted layer 5 pyramidal neurons account for the corticothalamic and corticotectal projection neurons.

Our observations further reinforce a clear dichotomy between talltufted and tall-simple pyramidal neurons. The presence of an apical dendritic tuft does not imply that these cells belong to a single type. The tall-simple cells are similar to short pyramids, in terms of both their local axonal arbors and their extrinsic projections, and they are distinctly different from tall-tufted pyramids. Furthermore, quantitative measures demonstrate that there is no overlap between tall-tufted and tall-simple neurons in either their total apical dendritic lengths or their numbers of apical dendritic branches. 
TALL-TUFTED TALL-SIMPLE

SHORT

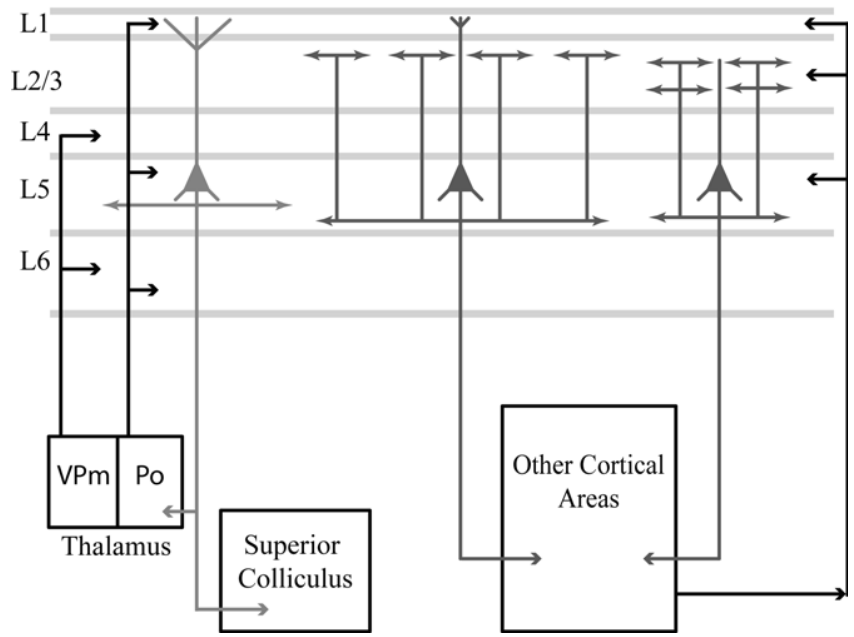

Figure 6. Schematic summarizes results and differences in morphologies, inputs, and projection targets between the three cell types in layer 5. The tall-tufted layer 5 pyramidal neurons project subcortically to both the thalamic nucleus Po and the superior colliculus, and only maintain local axonal arbors within the deep layers. This cell type receives extensive input within layer 1 , potentially from the thalamic nucleus $P$, corticocortical projections, and local inhibitory neurons. The tall-simple layer 5 pyramidal neurons project to other cortical areas and maintain local axons in both the superficial and deep layers. In addition, the axonal arborization within layer 2/3 appears to be patchy and extends farther in the horizontal direction. This cell type also receives input from layer 1, but significantly less than the tall-tufted layer 5 pyramidal neurons. The short layer 5 pyramidal neurons also project to other cortical areas and maintain local axons in both the superficial and deep layers in cortex, but unlike the tall-simple neurons' their superficial projection is more columnar in appearance. The short layer 5 pyramidal neurons are unable to receive input from layer 1 and most likely are integrating input from other cortical areas.

The differences in axonal and dendritic morphologies between the different projection neurons have important implications for the incorporation of the different cell types into the cortical circuit. Differences in dendritic arborization provide insight into possible differences in input to each cell type, while differences in axonal morphology provide insight into their differential outputs.

The most obvious difference between the dendritic arbors of the cell types we have observed is in layer 1 . Tall-tufted cells receive extensive input within layer 1 , tall-simple significantly less, and short cells can not receive any at all. This suggests that these cell types are likely to differ in their inputs from sources that target layer 1 . Most local excitatory connections do not preferentially target layer 1 and studies of functional input in brain slices have revealed that all layer 5 cell types studied receive local excitatory input from all cortical layers (layers 2-6) (Briggs and Callaway, 2005; Feldmeyer and Sakmann, 2000; Reyes and Sakmann, 1999; Schubert et al., 2001; Thomson and Deuchars, 1997). Therefore, these cells are likely to instead differ in the inputs they receive from distant excitatory sources and from local inhibitory neurons. Possible excitatory input sources which target layer 1 include thalamic inputs and corticocortical connections (Cauller et al., 1998; Cetas et al., 1999; Herkenham, 1980; Llinas et al., 2002; Lu and Lin, 1993; Mitchell and Cauller, 2001; Oda et al., 2004; Rockland and Pandya, 1979; Wise and Jones, 1976, 1978). Differential inhibition could come from either layer 1 inhibitory neurons (Anderson et al., 1992; Chu et al., 2003; Hestrin and Armstrong, 1996; Winer and Larue, 1989; Zhou and Hablitz, 1996) or from Martinotti cells located in deeper layers, both of which make synapses onto apical dendrites that extend into layer 1 (Silberberg and Markram, 2007;
Wang et al., 2004). For example, paired recordings have revealed that layer 5 Martinotti cells provide feedback inhibition onto tall-tufted layer 5 pyramids (Silberberg and Markram, 2007).

In the same way that dendritic morphology can highlight possible differences in inputs to different cell types, axonal morphology can highlight possible differences in their outputs. By retrogradely labeling cells of each type, we clearly revealed differences in outputs to subcortical structures vs. to the contralateral cortex. These differences proved to be tightly correlated with differences in the laminar specificity of local axonal arbors. Both short and tall-simple layer 5 pyramids make corticocortical connections and have local axons extending into the superficial layers, while tall-tufted pyramids project subcortically and restrict their local axons to deep layers.

Since the local axons of these cells can be well preserved in brain slices, many studies have already provided considerable insight into the local functional connections that are mediated by layer 5 pyramids. Our observations provide further insight into the cell types that could mediate these connections as well as how they correlate with extrinsic projections. Functional excitatory connections from layer 5 to layer $2 / 3$ must be contributed by short and tall-simple pyramids, but not by tall-tufted pyramids. Functional studies indicate that these projections to layer $2 / 3$ are likely to contact the distal apical dendrites of other layer 5 pyramidal neurons (Deuchars et al., 1994; Thomson and Deuchars, 1997), interneurons in layer 2/3 (Dantzker and Callaway, 2000), and layer 2/3 pyramidal neurons, though at a low frequency (Reyes and Sakmann, 1999; Shepherd and Svoboda, 2005; Shepherd et al., 2005; Thomson and Bannister, 1998). The tall-tufted layer 5 pyramids have been shown to synapse on the basal dendrites of other layer 5 pyramidal neurons (Feldmeyer and Sakmann, 2000; Markram, 1997; Markram et al., 1997; Schubert et al., 2001) and also onto layer 5 inhibitory neurons (Silberberg and Markram, 2007; Thomson and Deuchars, 1997).

Although tall-simple and short layer 5 pyramids have much in common, they are nevertheless different cell types. In addition to their definitive difference in apical dendritic morphology, our limited sample suggests a further difference between their local axonal arbors within layer $2 / 3$. Axons from the two short layer 5 pyramidal neurons which were reconstructed had a relatively columnar projection into layer $2 / 3$ (Figure 5). In contrast, the two tall-simple layer 5 pyramidal neurons had a patchier projection to layer $2 / 3$, covering a larger area laterally and in the anterior-posterior axis (Figure 5). This pattern was not noticed in our previous study of intracellularly labeled neurons in cortical slices, presumably due to cutting of axons during slice preparation. These differences in axonal arborization suggest that differences in input are correlated with differences in output and relate to differential functional roles for these cell types. For example, the longer lateral axons of tall-simple cells suggest that they may play a greater role in mediating interactions across barrel columns.

The anatomical observations we have made provide the clearest insight into identification of layer 5 pyramidal cell types and their possible connectivity. However, cell type specific differences in input sources and in outputs will ultimately interact with previously documented differences in intrinsic physiology (Contreras, 2004) as well as functional properties of synaptic connections (Feldmeyer and Sakmann, 2000) to establish the unique role of each cell type within the intact network.

\section{CONFLICT OF INTEREST STATEMENT}

The authors declare that the research was conducted in the absence of any commercial or financial relationships that could be construed as a potential conflict of interest.

\section{ACKNOWLEDGEMENTS}

We would like to thank Mauricio De La Parra for technical assistance. The work described here was supported by the National Institutes of Health EY010742 and MH063912 to EMC. 


\section{REFERENCES}

Akemann, W., Zhong, Y. M., Ichinohe, N., Rockland, K. S., and Knopfel, T. (2004) Transgenic mice expressing a fluorescent in vivo label in a distinct subpopulation of neocortical layer 5 pyramidal cells. J. Comp. Neurol. 480, 72-88.

Anderson, J. C., Martin, K. A., and Picanco-Diniz, C. W. (1992). The neurons in layer 1 of cat visual cortex. Proc. R. Soc. Lond., B, Biol. Sci. 248, 27-33.

Bourassa, J., Pinault, D., and Deschenes, M. (1995). Corticothalamic projections from the cortical barrel field to the somatosensory thalamus in rats: a single-fibre study using biocytin as an anterograde tracer. Eur. J. Neurosci. 7, 19-30.

Briggs, F., and Callaway, E. M. (2005). Laminar patterns of local excitatory input to layer 5 neurons in macaque primary visual cortex. Cereb. Cortex 15, 479-488.

Cauller, L. J., Clancy, B., and Connors, B. W. (1998). Backward cortical projections to primary somatosensory cortex in rats extend long horizontal axons in layer I. J. Comp. Neurol. 390, 297-310.

Cetas, J. S., de Venecia, R. K., and McMullen, N. T. (1999). Thalamocortical afferents of Lorente de No: medial geniculate axons that project to primary auditory cortex have collateral branches to layer I. Brain Res. 830, 203-208.

Chagnac-Amitai, Y., Luhmann, H. J., and Prince, D. A. (1990). Burst generating and regular spiking layer 5 pyramidal neurons of rat neocortex have different morphological features. J. Comp. Neurol. 296, 598-613.

Chu, Z., Galarreta, M., and Hestrin, S. (2003). Synaptic interactions of late-spiking neocortical neurons in layer 1. J. Neurosci. 23, 96-102.

Contreras, D. (2004). Electrophysiological classes of neocortical neurons. Neural Netw. $17,633-646$

Dantzker, J. L., and Callaway, E. M. (2000). Laminar sources of synaptic input to cortical inhibitory interneurons and pyramidal neurons. Nat. Neurosci. 3, 701-707.

Deschenes, M., Bourassa, J., and Pinault, D. (1994). Corticothalamic projections from layer V cells in rat are collaterals of long-range corticofugal axons. Brain Res. 664 215-219.

Deuchars, J., West, D. C., and Thomson, A. M. (1994). Relationships between morphology and physiology of pyramid-pyramid single axon connections in rat neocortex in vitro. J. Physiol. 478, 423-435.

Feldmeyer, D., and Sakmann, B. (2000). Synaptic efficacy and reliability of excitatory connections between the principal neurones of the input (layer 4) and output laye (layer 5) of the neocortex. J. Physiol. 525, 31-39.

Games, K. D., and Winer, J. A. (1988). Layer V in rat auditory cortex: projections to the inferior colliculus and contralateral cortex. Hear. Res. 34, 1-25.

Hallman, L. E., Schofield, B. R., and Lin, C. S. (1988). Dendritic morphology and axon collaterals of corticotectal, corticopontine, and callosal neurons in layer V of primary visual cortex of the hooded rat. J. Comp. Neurol. 272, 149-160.

Herkenham, M. (1980). Laminar organization of thalamic projections to the rat neocortex. Science 207, 532-535.

Hestrin, S., and Armstrong, W. E. (1996). Morphology and physiology of cortical neurons in layer I. J. Neurosci. 16, 5290-5300.

Hubener, M., and Bolz, J. (1988). Morphology of identified projection neurons in layer 5 of rat visual cortex. Neurosci. Lett. 94, 76-81.

Hubener, M., Schwarz, C., and Bolz, J. (1990). Morphological types of projection neurons in layer 5 of cat visual cortex. J. Comp. Neurol. 301, 655-674.

Katz, L. C. (1987). Local circuitry of identified projection neurons in cat visual cortex brain slices. J. Neurosci. 7, 1223-1249.

Killackey, H. P., Koralek, K. A., Chiaia, N. L., and Rhodes, R. W. (1989). Laminar and area differences in the origin of the subcortical projection neurons of the rat somatosensory cortex. J. Comp. Neurol. 282, 428-445.

Kim, H. G., and Connors, B. W. (1993). Apical dendrites of the neocortex: correlation between sodium- and calcium-dependent spiking and pyramidal cell morphology. J. Neurosci. 13, 5301-5311.

Koester, S. E., and O'Leary, D. D. (1992). Functional classes of cortical projection neurons develop dendritic distinctions by class-specific sculpting of an early common pattern. J. Neurosci. 12, 1382-1393.

Larkman, A., and Mason, A. (1990). Correlations between morphology and electrophysiology of pyramidal neurons in slices of rat visual cortex. I. Establishment of cell classes. J. Neurosci. 10, 1407-1414.

Larsen, D. D., and Callaway, E. M. (2006). Development of layer-specific axonal arborizations in mouse primary somatosensory cortex. J. Comp. Neurol. 494, 398-414.
Llinas, R. R., Leznik, E., and Urbano, F. J. (2002). Temporal binding via cortical coincidence detection of specific and nonspecific thalamocortical inputs: a voltagedependent dye-imaging study in mouse brain slices. Proc. Natl. Acad. Sci. U.S.A. 99, 449-454.

Lu, S. M., and Lin, R. C. (1993). Thalamic afferents of the rat barrel cortex: a light- and electron-microscopic study using Phaseolus vulgaris leucoagglutinin as an anterograde tracer. Somatosens. Mot. Res. 10,1-16.

Markram, H. (1997). A network of tufted layer 5 pyramidal neurons. Cereb. Cortex 7 , 523-533.

Markram, H., Lubke, J., Frotscher, M., Roth, A., and Sakmann, B. (1997). Physiology and anatomy of synaptic connections between thick tufted pyramidal neurones in the developing rat neocortex. J. Physiol. 500, 409-440.

Mitchell, B. D., and Cauller, L. J. (2001). Corticocortical and thalamocortical projections to layer I of the frontal neocortex in rats. Brain Res. 921, 68-77.

Oda, S., Kishi, K., Yang, J., Chen, S., Yokofujita, J., Igarashi, H., Tanihata, S., and Kuroda, M. (2004). Thalamocortical projection from the ventral posteromedial nucleus sends its collaterals to layer I of the primary somatosensory cortex in rat. Neurosci. Lett. 367, 394-398.

Reyes, A., and Sakmann, B. (1999). Developmental switch in the short-term modification of unitary EPSPs evoked in layer $2 / 3$ and layer 5 pyramidal neurons of rat neocortex. J. Neurosci. 19, 3827-3835.

Rockland, K. S., and Pandya, D. N. (1979). Laminar origins and terminations of cortical connections of the occipital lobe in the rhesus monkey. Brain Res. 179, 3-20.

Schofield, B. R., Hallman, L. E., and Lin, C. S. (1987). Morphology of corticotectal cells in the primary visual cortex of hooded rats. J. Comp. Neurol. 261, 85-97.

Schubert, D., Staiger, J. F., Cho, N., Kotter, R., Zilles, K., and Luhmann, H. J. (2001). Layer-specific intracolumnar and transcolumnar functional connectivity of layer $\mathrm{V}$ pyramidal cells in rat barrel cortex. J. Neurosci. 21, 3580-3592.

Shepherd, G. M., Stepanyants, A., Bureau, I., Chklovskii, D., and Svoboda, K. (2005). Geometric and functional organization of cortical circuits. Nat. Neurosci. 8 782-790.

Shepherd, G. M., and Svoboda, K. (2005). Laminar and columnar organization of ascending excitatory projections to layer $2 / 3$ pyramidal neurons in rat barrel cortex J. Neurosci. 25, 5670-5679.

Silberberg, G., and Markram, H. (2007). Disynaptic inhibition between neocortical pyramidal cells mediated by Martinotti cells. Neuron 53, 735-746.

Thomson, A. M., and Bannister, A. P. (1998). Postsynaptic pyramidal target selection by descending layer III pyramidal axons: dual intracellular recordings and biocytin filling in slices of rat neocortex. Neuroscience 84, 669-683.

Thomson, A. M., and Deuchars, J. (1997). Synaptic interactions in neocortical local circuits: dual intracellular recordings in vitro. Cereb. Cortex 7, 510-522.

Tsiola, A., Hamzei-Sichani, F., Peterlin, Z., and Yuste, R. (2003). Quantitative morphologic classification of layer 5 neurons from mouse primary visual cortex. J. Comp. Neurol. $461,415-428$

Veinante, P., Lavallee, P., and Deschenes, M. (2000). Corticothalamic projections from layer 5 of the vibrissal barrel cortex in the rat. J. Comp. Neurol. 424, 197-204.

Wang, Z., and McCormick, D. A. (1993). Control of firing mode of corticotectal and corticopontine layer $\mathrm{V}$ burst-generating neurons by norepinephrine, acetylcholine, and 1S,3R-ACPD. J. Neurosci. 13, 2199-2216.

Wang, Y., Toledo-Rodriguez, M., Gupta, A., Wu, C., Silberberg, G., Luo, J., and Markram, H. (2004). Anatomical, physiological and molecular properties of Martinotti cells in the somatosensory cortex of the juvenile rat. J. Physiol. 561, 65-90.

Wickersham, I. R., Finke, S., Conzelmann, K. K., and Callaway, E. M. (2007). Retrograde neuronal tracing with a deletion-mutant rabies virus. Nat. Methods 4, 47-49.

Winer, J. A., and Larue, D. T. (1989). Populations of GABAergic neurons and axons in layer I of rat auditory cortex. Neuroscience 33, 499-515.

Wise, S. P., and Jones, E. G. (1976). The organization and postnatal development of the commissural projection of the rat somatic sensory cortex. J. Comp. Neurol. 168, 313-343.

Wise, S. P., and Jones, E. G. (1977). Somatotopic and columnar organization in the corticotectal projection of the rat somatic sensory cortex. Brain Res. 133, 223-235.

Wise, S. P., and Jones, E. G. (1978). Developmental studies of thalamocortical and commissural connections in the rat somatic sensory cortex. J. Comp. Neurol. 178, 187-208.

Zhou, F. M., and Hablitz, J. J. (1996). Morphological properties of intracellularly labeled layer I neurons in rat neocortex. J. Comp. Neurol. 376, 198-213. 